Mathematical Modelling and Analysis

Volume 22 Number 6, November 2017, 763-784

https://doi.org/10.3846/13926292.2017.1376295

(c) Vilnius Gediminas Technical University, 2017
Publisher: Taylor\&Francis and VGTU

http://www.tandfonline.com/TMMA

ISSN: $1392-6292$

eISSN: $1648-3510$

\title{
Boundary Control and Stabilization of an Axially Moving Viscoelastic String under a Boundary Disturbance
}

\section{Abdelkarim Kelleche}

Université Djilali Bounâama, Faculté des Sciences et de la Technologie

Route Theniet El Had, Soufay 44225 Khemis Miliana, Algeria

E-mail: a.kelleche@univ-dbkm.dz

Received March 15, 2017; revised August 31, 2017; published online November 15, 2017

\begin{abstract}
In this paper, we consider a system modelling an axially moving viscoelastic string subject to an unknown boundary disturbance. It is controlled by a hydraulic touch-roll actuator at the right boundary which is capable of suppressing the transverse vibrations that occur during the movement of the string. The multiplier method is employed to design a robust boundary control law to ensure the reduction of the transvesre vibrations of the string.
\end{abstract}

Keywords: axially moving string, dynamic actuator, viscoelasticity, boundary control.

AMS Subject Classification: 35L20; 65N30; 74G25; 74G30; 93D15; 93D20.

\section{Introduction}

We consider an axially moving viscoelastic string subject to vibrations and an unknown boundary disturbance. The string is moving in the direction of its axis with a constant speed $c$. The left boundary is assumed fixed in the sense that there is no vertical movement but it allows the string to move in the horizontal direction. A controller mechanism (dynamic actuator) is attached at the right boundary. The problem is modeled as a system composed of a partial integro-differential equation describing the movement of the string and an integro-ordinary differential equation describing the actuator dynamics. The system may be written as

$$
\left\{\begin{array}{l}
\rho y_{t t}+2 \rho c y_{x t}+\left(\rho c^{2}-T_{s}\right) y_{x x}+\int_{0}^{t} h(t-s)\left(a(x) y_{x}(s)\right)_{x} d s=0 \\
x \in(0, l), t>0, \quad y(0, t)=0, t \geq 0 \\
F_{c}(t)=m y_{t t}(l, t)+\left(\eta_{m}-\rho c\right) y_{t}(l, t)+\left(T_{s}-\rho c^{2}\right) y_{x}(l, t) \\
-a(l) \int_{0}^{t} h(t-s) y_{x}(l, s) d s+d(t), t \geq 0
\end{array}\right.
$$


where $y(x, t)$ is the transversal displacement of the string at the position $x$ and at time $t ; y(l, t)$ indicates the position actuator, where $l$ is the length of the controlled part of the string; $\rho>0$ denotes the mass per unit length; $m$ and $\eta_{m}$ denote the mass and the damping coefficient of the actuator, respectively; $F_{c}(t)$ is a control force applied by the dynamic actuator to suppress the transverse vibrations; $d(t)$ denotes the unknown disturbance force exerted on the actuator due to the transverse vibration of the string; $T_{s}$ is a constant axial tension of the string.

The convolution term, also called viscoelastic damping term, in the equation (1.1), describes the relationship between the stress and the history of the strain in the string, according to Boltzmann theory. The function $h$ represents the kernel of the memory term or the relaxation function. For more details about the physical meaning, see $[6,7,10]$. Because the string travels at constant speed $c$, the total derivative operator with respect to time is defined by

$$
\frac{d}{d t}=\frac{\partial}{\partial t}+c \frac{\partial}{\partial x} .
$$

The system (1.1), without viscoelastic term, can be derived using the generalized Hamilton's principle (see [33]). In case the string is made of a viscoelastic material, we recall the equation of linear viscoelasticity in one dimensional space (see $[8,9])$

$$
\frac{d}{d t}\left[\rho(x) \frac{d}{d t} y(x, t)\right]=\left[C(x, t) y_{x}(x, t)-\int_{0}^{t} H(x, t-s) y_{x}(x, s) d s\right]_{x},
$$

which is derived from the constitutive relationship between the stress and the strain

$$
\sigma(t)=E \epsilon(t)-E \int_{0}^{t} h(t-s) \epsilon(s) d s
$$

with $\epsilon(x, t)=y(x, t)$, where $\sigma$ and $\epsilon$ are respectively, the stress and the strain and $E$ is Young's modulus (see [26] for more details). Note, we consider here the case where $\rho, C$ are constants and $H(x, t)=a(x) h(t), x \in[0, l], t \geq 0$. The first term in (1.3) is evaluated using (1.2) as follows

$$
\rho \frac{d^{2}}{d t} y(x, t)=\rho\left(y_{t t}(x, t)+2 c y_{x t}(x, t)+c^{2} y_{x x}(x, t)\right) .
$$

Considering (1.4) in (1.3) and taking into account the previous considerations, the Equation (1.3) takes the form of the main equation in (1.1). Note that the right boundary condition in (1.1) is an ODE that describes the motion of the hydraulic actuator in compliance with the transversal force at $x=l$.

During the last decades, the interest for axially moving systems has increased considerably. In fact many problems in engineering consist of axially moving structures such as magnetic tapes, steel strips, band saws, chains, power transmission chains and belts (see [1], [25]). An axially moving system may be a string, a beam or a belt equation. An important issue in industry is how to control the unwanted vibrations. These vibrations are the result of several 
factors, most notably: material non-uniformity, environmental disturbances, high speed and the manufacturing process.

Several means have been discussed in order to reduce or to put an end to these vibrations. Boundary control is one of the efficient ways adopted so far. Many papers have been published in this regard. We cite here only few of them. In the case of moving strings: one may consult $[5,11,21,29])$. In the case of moving beams we refer the reader to $[17,18,33]$ and in the case of moving belts, we mention the work in [23]. The stabilization using boundary control of viscoelastic type was also investigated in $[15,16,19]$. The authors obtained an uniform decay result under some conditions on the relaxation function.

In particular, the authors in [12] considered a system describing an axially moving string and a mass-damper-spring (MDS) controller applied at the right hand side boundary of the string. The governing equation and boundary conditions are obtained by using Hamilton's principle

$$
\left\{\begin{array}{l}
\rho y_{t t}+2 \rho c y_{x t}+\left(\rho c^{2}-T_{s}\right) y_{x x}+c_{v}\left(y_{t}+c y_{x}\right)=0, x \in(0, l), t>0 \\
y(0, t)=0, t \geq 0 \\
F_{c}=m y_{t t}(l, t)+\left(d_{m}-\rho c\right) y_{t}(l, t)+k_{m} y(l, t)+\left(T_{s}-\rho c^{2}\right) y_{x}(l, t)
\end{array}\right.
$$

where $m, d_{m}$ and $k_{m}$ denote the lump mass, the viscous damper coefficient and the stiffness coefficient of the spring, respectively. The authors proved that the system is exponentially stable by using the $C_{0}$ semigroup theory under the following feedback control law

$$
f_{c}(t)=-\alpha y_{t}(l, t), \alpha>0, t>0 .
$$

The authors in [33], considered a system describing an axially moving string under a spatiotemporally varying tension. The system is divided into two parts: a controlled span and an uncontrolled span. A hydraulic touch roll actuator is placed in the middle of the string. The governing equation and the boundary conditions are obtained by using Hamilton's principle

$$
\left\{\begin{array}{l}
\rho y_{t t}+2 \rho c y_{x t}+\rho c^{2} y_{x x}-\left(T_{s}(x, t) y_{x}\right)_{x}+c_{v}\left(y_{t}+c y_{x}\right)=0 \\
x \in(0, l), t>0, \quad y(0, t)=0, t \geq 0 \\
F_{c}(t)=m y_{t t}(l, t)+\left(\eta_{m}-\rho c\right) y_{t}(l, t)+\left(T_{s}(l, t)-\rho c^{2}\right) y_{x}(l, t),
\end{array}\right.
$$

where $c_{v}$ denotes the damping coefficient in the string. The authors proved the asymptotic stability of the closed loop system under the robust boundary control scheme by using the semigroup theory provided that the lower bound of the tension $T_{s}(x, t)$ is sufficiently bigger than the derivatives of tension $\left(T_{s}(x, t)\right)_{t}$ and $\left(T_{s}(x, t)\right)_{x}$.

Our goal throughout this work is to achieve the stability of the problem (1.1) under an appropriate boundary controller. We shall rely on the damping property enjoyed by viscoelastic materials rather than the frictional damping used in (1.5) and (1.6). Viscoelastic materials are present in many fields of engineering. They provide an efficient mechanism of dissipation of mechanical 
vibrations into heat. Consequently, they are capable of reducing vibrations and hence improving the lifespan of the structures.

In this work, unlike most of the published articles in viscoelasticity where the relaxation functions were assumed to be strictly decreasing and with a certain rate, see for example $[2,27]$, we consider relaxation functions that may have null derivatives on some subsets of $(0, \infty)$, this kind of kernels was investigated in $([30]-[32])$ and $[27]$.

The situation here is different from the ones in these papers and many others where the structures were not moving. Indeed, the new feature here is the treatment of a string which is moving (in addition to vibrating). Unlike the situations where the strings are motionless, in our case here, the spatial variable $x$ varies with time. This will affect the derivative of the energy and gives rise to new boundary terms whose handling is often challenging.

The rest of this paper is organized as follows: in the next section, we prepare some material needed in the proof of our result, like some lemmas (Poincaré's inequality, Young's inequality) and some useful notation. We introduce the different functionals by which we modify the classical energy to get an equivalent useful one. We also determine the control force $F_{c}(t)$ that will act on the right endpoint of the string. In section 3 , we start by introducing some further notation which will be used repeatedly in the proof and formulating the assumptions on the relaxation function, then we provide the proof of our result which relies on the multiplier method.

\section{Preliminaries}

In this section, we introduce the following mathematical preliminaries: some definitions and notation, useful technical lemmas which will be widely used throughout this paper. For every measurable set $\mathcal{A} \subset \mathbb{R}_{+}$, we define for all $t \geq 0$

$$
\hat{h}(\mathcal{A})=\frac{1}{k} \int_{\mathcal{A}} h(s) d s,
$$

where $k=\int_{0}^{\infty} h(s) d s$ and $\mathcal{A}_{t}=\mathcal{A} \cap[0, t]$. The flatness set and the the flatness rate of $h$ are defined by

$$
\mathcal{F}_{h}=\left\{s \in \mathbb{R}_{+}: h(s)>0 \text { and } h^{\prime}(s)=0\right\}, \quad \mathcal{R}_{h}=\hat{h}\left(\mathcal{F}_{h}\right),
$$

respectively. We also define

$$
\tilde{F}_{h t}=\left\{s \in \mathbb{R}_{+}: 0 \leq s \leq t, h(t-s)>0 \text { and } h^{\prime}(t-s)=0\right\} .
$$

For the function $a(x)$ and for the relaxation function $h(t)$, we formulate the following assumptions

(A1) $h(t) \geq 0$ for all $t \geq 0$ and $h^{\prime}(t) \leq 0$ for almost all $t>0$.

(A2) $a:[0, l] \longrightarrow \mathbb{R}_{+}$is a nonnegative bounded function and $a(x) \geq a_{0}>0$ on $[0, l]$ with

$$
T_{s}-\rho c^{2}-\|a\|_{\infty} \int_{0}^{\infty} h(s) d s=: T_{s}-\rho c^{2}-\|a\|_{\infty} k>0 .
$$


(A3) There exists $\lambda>0$ such that $\int_{0}^{\infty} h(s) e^{\lambda s} d s<\infty$.

(A4) The disturbance function $d(t)$ is uniformly bounded, i.e., $|d(t)| \leq \mu_{d}$, where $\mu_{d}$ is an unknown positive constant.

Let $t_{*}>0$ be a number such that $\int_{0}^{t_{*}} h(s) d s=h_{*}>0$. For simplicity, we consider kernels continuous everywhere and continuously differentiable a.e. Throughout this paper, we denote by $\circ$ the operator, defined by

$(h \circ v)(x, t)=\int_{0}^{t} h(t-s) a(x)|v(x, t)-v(x, s)|^{2} d s, v(x,.) \in L^{\infty}(0, \infty), t \geq 0$

and by $\|$.$\| the L^{2}$-norm.

Example 1. Let $h(t)$ a non-increasing kernel defined by

$$
h(t)= \begin{cases}e^{-\delta t}, & 0 \leq t \leq a_{1} \\ C=e^{-\delta a_{1}}, & a_{1} \leq t \leq a_{2} \\ e^{-\delta a_{1} t / a_{2}}, & t \geq a_{2}\end{cases}
$$

with

$$
0<a_{1}<1, \delta>1 /\left(1-a_{1}\right), \quad 0<a_{1}<a_{2} \leq \delta a_{1} /\left(1+\delta a_{1}\right), \quad \mathcal{F}_{h}=\left[a_{1}, a_{2}\right] .
$$

A simple computation shows that $h(t)$ satisfies the hypotheses (A1)-(A2) and satisfy the hypothesis (A3) for $\lambda<\delta a_{1} / a_{2}$.

In the sequel, we give some lemmas which will be useful in the proof of our result.

Lemma 1. (See [13]) Let $\Phi(x, t) \in C\left(\mathbb{R}_{+} ; H^{1}(0, l)\right)$ satisfying the boundary condition

$$
\Phi(0, t)=0, \forall t \in[0, \infty),
$$

then the following inequalities hold

$$
\begin{aligned}
& \Phi^{2}(x, t) \leq l\left\|\Phi_{x}\right\|^{2}, \forall t \in[0, \infty), \forall x \in[0, l], \\
& \|\Phi\|^{2} \leq l^{2}\left\|\Phi_{x}\right\|^{2}, \forall t \in[0, \infty) .
\end{aligned}
$$

Lemma 2. (Young's inequality) Let $f \in L^{p}(\mathbb{R})$ and $g \in L^{q}(\mathbb{R})$ with $1 \leq p, q \leq$ $\infty$ and $\frac{1}{r}=\frac{1}{p}+\frac{1}{q}$. Then $f * g \in L^{r}(\mathbb{R})$ and

$$
\|f * g\|_{L^{r}} \leq\|f\|_{L^{p}}\|g\|_{L^{q}} .
$$

Lemma 3. (See [32]) We have for $g \in C(0, \infty)$ and $v \in C\left((0, \infty) ; L^{2}(0, l)\right)$

$$
\begin{aligned}
\int_{0}^{l} a(x) v(t) \int_{0}^{t} g(t-s) v(s) d s d x=\frac{1}{2}\left(\int_{0}^{t} g(s) d s\right)\|\sqrt{a(x)} v\|^{2} \\
\quad+\frac{1}{2} \int_{0}^{t} g(t-s)\|\sqrt{a(x)} v(s)\|^{2} d s-\frac{1}{2} \int_{0}^{l}(g \circ v) d x, \quad t \geq 0 .
\end{aligned}
$$


Lemma 4. ([31]) Suppose that $g$ is a nonnegative function such that $e^{\lambda t} g(t) \in$ $L^{1}(0, \infty)$ for some $\lambda>0$. Then, for any $\epsilon>0$, there exists $0<\sigma<\lambda$ such that

$$
\int_{0}^{t} g(s) e^{2 \sigma s} d s \leq(1+\epsilon) \int_{0}^{\infty} g(s) d s, t \geq 0 .
$$

The following inequality will be used repeatedly throughout this paper.

Lemma 5. (Cauchy inequality, see [13]) For $a, b \in \mathbb{R}$, the following inequality holds

$$
a b \leq \delta a^{2}+\frac{b^{2}}{4 \delta}, \delta>0 .
$$

In order to state the existence result of the system (1.1). Let we consider the usual Hilbert space $L^{2}(0, l)$ with the inner product $(.,$.$) and the inner product$ induced norm $\|$.$\| . We also define$

$$
\mathcal{V}=\left\{v \in H^{1}(0, l), v(0)=0\right\}
$$

equipped with norm of $H^{1}(0, l)$.

Theorem 1. Let $\left(y_{0}, y_{1}\right) \in\left(\mathcal{V} \cap H^{2}(0, l)\right) \times \mathcal{V}$ and let $T>0$, then there exists a unique global solution to problem (1.1) such that

$y \in L^{\infty}\left([0, T), \mathcal{V} \cap H^{2}(0, l)\right), y_{t} \in L^{\infty}([0, T), \mathcal{V}), y_{t t} \in L^{2}\left([0, T), L^{2}(0, l)\right)$.

Moreover, we havey $\in C([0, T), \mathcal{V}), y_{t} \in C\left([0, T), L^{2}(0, l)\right)$.

Proof. The proof is based on the Galerkin approximation method. For this, we refer the reader to $[3,4,14]$.

\section{Control design and asymptotic behavior}

In this section, we state and prove our main result. We first modify the classical energy of the system (1.1) as follows

$$
\begin{aligned}
E(t)= & \frac{\rho}{2}\left\|y_{t}\right\|^{2}+\frac{1}{2}\left(T_{s}-\rho c^{2}\right)\left\|y_{x}\right\|^{2}-\frac{1}{2}\left(\int_{0}^{t} h(s) d s\right)\left\|\sqrt{a(x)} y_{x}\right\|^{2} \\
& +\frac{1}{2} \int_{0}^{l}\left(h \circ y_{x}\right) d x+\frac{m}{2}\left(y_{t}(l)+c y_{x}(l)\right)^{2}+\frac{1}{2 \gamma_{d}} \tilde{\mu}_{d}^{2}, t \geq 0,
\end{aligned}
$$

where $\gamma_{d}>0, \tilde{\mu}_{d}=\hat{\mu}_{d}-\mu_{d}$ and $\hat{\mu}_{d}$ is the adaptive estimate of $\mu_{d}$ which will be specified in the sequel. Therefore,

$$
\begin{aligned}
& \frac{d}{d t} E(t)=\int_{0}^{l} \frac{d}{d t} \widetilde{E}(x, t) d x+\frac{1}{2} \frac{d}{d t}\left[m\left(y_{t}(l)+c y_{x}(l)\right)^{2}+\frac{1}{\gamma_{d}} \tilde{\mu}_{d}^{2}\right] \\
= & \int_{0}^{l}\left(\frac{\partial}{\partial t} \widetilde{E}(x, t)+c \frac{\partial}{\partial x} \widetilde{E}(x, t)\right) d x+\frac{1}{2} \frac{d}{d t}\left[m\left(y_{t}(l)+c y_{x}(l)\right)^{2}+\frac{1}{\gamma_{d}} \tilde{\mu}_{d}^{2}\right] \\
= & \int_{0}^{l} \frac{\partial}{\partial t} \widetilde{E}(x, t) d x+\left.c \widetilde{E}(x, t)\right|_{0} ^{l}+\frac{1}{2} \frac{d}{d t}\left[m\left(y_{t}(l)+c y_{x}(l)\right)^{2}+\frac{1}{\gamma_{d}} \tilde{\mu}_{d}^{2}\right], t \geq 0,
\end{aligned}
$$


where

$\widetilde{E}(x, t)=\frac{\rho}{2} y_{t}^{2}(x, t)+\frac{1}{2}\left(T_{s}-\rho c^{2}-a(x) \int_{0}^{t} h(s) d s\right) y_{x}^{2}(x, t)+\frac{1}{2}\left(h \circ y_{x}\right)(x, t)$

for $x \in[0, l]$ and $t \geq 0$. That is, the rate of change (of mass) is equal to the sum of the rate of the accumulation of mass and the net rate of flow across the surface (here boundary) (see $[20,28]$ ). Back to $(3.1)$, the total derivative of $E(t)$ is equal to

$$
\begin{aligned}
\frac{d}{d t} E(t)= & \rho \int_{0}^{l} y_{t} y_{t t} d x+\left(T_{s}-\rho c^{2}\right) \int_{0}^{l} y_{x} y_{x t} d x \\
& -\left(\int_{0}^{t} h(s) d s\right) \int_{0}^{l} a(x) y_{x} y_{x t} d x-\frac{1}{2} h(t)\left\|\sqrt{a(x)} y_{x}\right\|^{2} \\
& +\frac{1}{2} \int_{0}^{l}\left(h^{\prime} \circ y_{x}\right) d x+\int_{0}^{l} y_{x t} \int_{0}^{t} h(t-s)\left(y_{x}(t)-y_{x}(s)\right) d s d x \\
& +\left.c \widetilde{E}(x, t)\right|_{0} ^{l}+m\left(y_{t}+c y_{x}\right)(l)\left(y_{t t}+c y_{x t}\right)(l)+\frac{1}{\gamma_{d}} \tilde{\mu}_{d} \frac{d}{d t} \hat{\mu}_{d}, t \geq 0 .
\end{aligned}
$$

In view of the definition of $\widetilde{E}(x, t)$ and taking into account the boundary conditions in (1.1), we see that for $t \geq 0$

$$
\begin{aligned}
\left.\widetilde{E}(x, t)\right|_{0} ^{l} \leq & \frac{\rho}{2} y_{t}^{2}(l)+\frac{1}{2}\left(T_{s}-\rho c^{2}-a(l) \int_{0}^{t} h(s) d s\right) y_{x}^{2}(l)+\frac{1}{2}\left(h \circ y_{x}\right)(l) \\
\leq & \frac{\rho}{2} y_{t}^{2}(l)+\frac{1}{2}\left(T_{s}-\rho c^{2}\right) y_{x}^{2}(l)-a(l) y_{x}(l) \int_{0}^{t} h(t-s) y_{x}(l, s) d s \\
& +\frac{a(l)}{2} \int_{0}^{t} h(t-s) y_{x}^{2}(l, s) d s
\end{aligned}
$$

Substituting $y_{t t}$ and $y_{t t}(l)$ from Equation(1.1) into Equation(3.2), taking into account the relation (3.3) and integrating by parts we find

$$
\begin{aligned}
\frac{d}{d t} E(t) & \leq-\frac{\rho c}{2} y_{t}^{2}(l)-\frac{1}{2} h(t)\left\|\sqrt{a(x)} y_{x}\right\|^{2}+\frac{1}{2} \int_{0}^{l}\left(h^{\prime} \circ y_{x}\right) d x \\
& -\frac{c}{2}\left(T_{s}-\rho c^{2}\right) y_{x}^{2}(l)+\frac{c a(l)}{2} \int_{0}^{t} h(t-s) y_{x}^{2}(l, s) d s+\left(y_{t}+c y_{x}\right)(l)\left[F_{c}(t)\right. \\
& \left.-\left(\eta_{m}-\rho c\right) y_{t}(l)+m c y_{x t}(l)-d(t)\right]+\frac{1}{\gamma_{d}} \tilde{\mu}_{d} \frac{d}{d t} \hat{\mu}_{d}, t \geq 0
\end{aligned}
$$

Now, the robust control law for the right boundary control force $F_{c}(t)$ is then proposed as follows

$$
F_{c}(t)=\left(\eta_{m}-\rho c\right) y_{t}(l)-m c y_{x t}(l)+F_{d}(t), t \geq 0 .
$$

The extra term $F_{d}(t)$ is considered as a new input signal determined as based on robust control strategy (see [34]) and is given by

$$
F_{d}(t)=-\frac{\hat{\mu}_{d}^{2}(t)}{\hat{\mu}_{d}(t)|\bar{y}(l)|+\varepsilon_{d}} \bar{y}(l),
$$


where $\varepsilon_{d}>0$ and $\bar{y}$ is a function that will be determined later. The adaptation law $\hat{\mu}_{d}$ is proposed as

$$
\frac{d}{d t} \hat{\mu}_{d}(t)=-\delta_{d} \hat{\mu}_{d}(t)+\gamma_{d}|\bar{y}(l)|, \quad \delta_{d}>0 .
$$

Remark 1. The measurement of the velocity $y_{t}$, slope $y_{x}$ and slope rate $y_{x t}$ at the endpoint $x=l$ are required to implement the boundary force control law (3.5). By using an encoder (or photodiode) on the actuator and two laser sensors, the actuator displacement $y(l)$ and the slope $y_{x}(l)$ can be measured, respectively (see $[22]$ ). Then, the velocity $y_{t}(l)$ and the slope rate $y_{x t}(l)$ can be implemented by backward differentiation of signals that measures $y(l)$ and $y_{x}(l)$, respectively.

Considering the expression (3.5), (3.4) turns into

$$
\begin{aligned}
\frac{d}{d t} E(t) \leq & -\frac{\rho c}{2} y_{t}^{2}(l)-\frac{1}{2} h(t)\left\|\sqrt{a(x)} y_{x}\right\|^{2}+\frac{1}{2} \int_{0}^{l}\left(h^{\prime} \circ y_{x}\right) d x \\
& -\frac{c}{2}\left(T_{s}-\rho c^{2}\right) y_{x}^{2}(l)+\frac{c a(l)}{2} \int_{0}^{t} h(t-s) y_{x}^{2}(l, s) d s \\
& +\left(y_{t}+c y_{x}\right)(l)\left(F_{d}(t)-d(t)\right)+\frac{1}{\gamma_{d}} \tilde{\mu}_{d} \frac{d}{d t} \hat{\mu}_{d} .
\end{aligned}
$$

Next, we define the functionals

$$
\begin{aligned}
\Phi_{1}(t)= & \rho \int_{0}^{l} y y_{t} d x+m y(l)\left(y_{t}+c y_{x}\right)(l), \quad t \geq 0, \\
\Phi_{2}(t)=- & \rho \int_{0}^{l} y_{t} \int_{0}^{t} h(t-s)(y(t)-y(s)) d s d x \\
& \quad-m\left(y_{t}+c y_{x}\right)(l) \int_{0}^{t} h(t-s)(y(l, t)-y(l, s)) d s, t \geq 0 .
\end{aligned}
$$

The modified functional we will utilize is

$$
L(t)=E(t)+\sum_{i=1}^{2} \lambda_{i} \Phi_{i}(t), t \geq 0,
$$

for some $\lambda_{i}>0, i=1,2$ to be determined. The first result shows that $L(t)$ and $E(t)$ are equivalent.

Proposition 1. We have for each $t>0$ and small $\lambda_{i}>0, i=1,2$

$$
\frac{1}{2} E(t) \leq L(t) \leq 2 E(t)
$$

Proof. Using Lemma 5 and Lemma 1, we have

$$
\begin{aligned}
\Phi_{1}(t) & \leq \frac{\rho}{2}\left(\left\|y_{t}\right\|^{2}+\|y\|^{2}\right)+\frac{m}{2}\left[y^{2}(l)+\left(y_{t}+c y_{x}\right)^{2}(l)\right] \\
& \leq \frac{\rho}{2}\left\|y_{t}\right\|^{2}+\frac{l}{2}(\rho l+m)\left\|y_{x}\right\|^{2}+\frac{m}{2}\left(y_{t}+c y_{x}\right)^{2}(l), t \geq 0 .
\end{aligned}
$$


Similarly, we get

$$
\Phi_{2}(t) \leq \frac{\rho}{2}\left\|y_{t}\right\|^{2}+\frac{k l}{2 a_{0}}(\rho l+m) \int_{0}^{l}\left(h \circ y_{x}\right) d x+\frac{m}{2}\left(y_{t}(l)+c y_{x}(l)\right)^{2}, t \geq 0 .
$$

Therefore

$$
\begin{aligned}
L(t) & \leq \frac{\rho}{2}\left(1+\lambda_{1}+\lambda_{2}\right)\left\|y_{t}\right\|^{2}+\frac{1}{2}\left[\left(T_{s}-\rho c^{2}\right)+\lambda_{1} l(\rho l+m)\right]\left\|y_{x}\right\|^{2} \\
& -\frac{1}{2}\left(\int_{0}^{t} h(s) d s\right)\left\|\sqrt{a(x)} y_{x}\right\|^{2}+\frac{1}{2}\left[1+\frac{\lambda_{2} k l}{a_{0}}(\rho l+m)\right] \int_{0}^{l}\left(h \circ y_{x}\right) d x \\
& +\frac{m}{2}\left(1+\lambda_{1}+\lambda_{2}\right)\left(y_{t}+c y_{x}\right)^{2}(l), t \geq 0 .
\end{aligned}
$$

On the other hand for $t \geq 0$

$$
\begin{aligned}
& 2 E(t)-L(t) \geq \frac{\rho}{2}\left(1-\lambda_{1}-\lambda_{2}\right)\left\|y_{t}\right\|^{2} \\
& \quad+\frac{1}{2}\left[\left(T_{s}-\rho c^{2}\right)-\|a\|_{\infty} k-\lambda_{1} l(\rho l+m)\right]\left\|y_{x}\right\|^{2} \\
& \quad+\frac{1}{2}\left[1-\frac{\lambda_{2} k l}{a_{0}}(\rho l+m)\right] \int_{0}^{l}\left(h \circ y_{x}\right) d x+\frac{m}{2}\left(1-\lambda_{1}-\lambda_{2}\right)\left(y_{t}+c y_{x}\right)^{2}(l) .
\end{aligned}
$$

If we choose $\lambda_{1}<\min \left\{1,\left[\left(T_{s}-\rho c^{2}\right)-\|a\|_{\infty} k\right] /[l(\rho l+m)]\right\}$ and $\lambda_{2}<$ $\min \left\{1\left[\frac{k l}{a_{0}}(\rho l+m)\right], 1-\lambda_{1}\right\}$, then $2 E(t)-L(t) \geq 0, t \geq 0$. Similar computations and same arguments yield $L(t)-\frac{1}{2} E(t) \geq 0, t \geq 0$.

Theorem 2. Assume that the hypotheses (A1)-(A4) hold. If $\mathcal{R}_{h}$ is small enough, then there exist positive constants $C, \gamma$ and sufficiently small $\epsilon$ such that

$$
E(t) \leq C e^{-\gamma t}+\epsilon, t \geq 0 .
$$

In case where the disturbance function $d(t)=0$, then there exist positive constants $K$ and $\tau$ such that

$$
E(t) \leq K e^{-\tau t}, t \geq 0
$$

Proof. The total derivative of $\Phi_{1}(t)$ is given by (note here again that we are taking into account the axial motion of the string)

$$
\begin{aligned}
\frac{d}{d t} \Phi_{1}(t) & =\int_{0}^{l} \frac{d}{d t} \widetilde{\Phi_{1}}(x, t) d x+m \frac{d}{d t}\left[y(l)\left(y_{t}+c y_{x}\right)(l)\right] \\
& =\int_{0}^{l}\left(\frac{\partial}{\partial t} \widetilde{\Phi_{1}}(x, t)\right) d x+\left.c \widetilde{\Phi_{1}}(x, t)\right|_{0} ^{l}+m \frac{d}{d t}\left[y\left(y_{t}+c y_{x}\right)\right](l), t \geq 0
\end{aligned}
$$

where

$$
\widetilde{\Phi_{1}}(x, t)=\rho y(x, t) y_{t}(x, t), x \in[0, l], t \geq 0 .
$$


This definition together with the boundary condition in (1.1) gives

$$
\left.\widetilde{\Phi_{1}}(x, t)\right|_{0} ^{l}=\rho y(l) y_{t}(l), t \geq 0 .
$$

Using (1.1) together with (3.9) and (3.10), we entail

$$
\begin{aligned}
& \frac{d}{d t} \Phi_{1}(t)=\rho\left\|y_{t}\right\|^{2}-\int_{0}^{l} y\left[2 \rho c y_{x t}+\left(\rho c^{2}-T_{s}\right) y_{x x}\right] d x \\
& \quad-\int_{0}^{l} y \int_{0}^{t} h(t-s)\left(a(x) y_{x}(s)\right)_{x} d s d x+y(l)\left[F_{c}(t)-\left(\eta_{m}-\rho c\right) y_{t}(l)\right. \\
& \left.\quad-\left(T_{s}-\rho c^{2}\right) y_{x}(l)\right]+y(l)\left[a(1) \int_{0}^{t} h(t-s) y_{x}(l, s) d s+m c y_{x t}(l)-d(t)\right] \\
& \quad+m y_{t}(l)\left(y_{t}+c y_{x}\right)(l)+\rho c y(l) y_{t}(l), t \geq 0
\end{aligned}
$$

Next, taking into account the boundary conditions in (1.1) and the expression (3.5), the identity (3.11) becomes

$$
\begin{aligned}
\frac{d}{d t} \Phi_{1}(t)= & \rho\left\|y_{t}\right\|^{2}-\left(T_{s}-\rho c^{2}\right)\left\|y_{x}\right\|^{2}+2 \rho c \int_{0}^{l} y_{t} y_{x} d x \\
& +\int_{0}^{l} y_{x} \int_{0}^{t} h(t-s) a(x) y_{x}(s) d s d x \\
& +y(l)\left(F_{d}(t)-d(t)\right)-\rho c y(l) y_{t}(l)+m y_{t}\left(y_{t}+c y_{x}\right)(l), t \geq 0 .
\end{aligned}
$$

The third term in the right hand side of (3.12) is estimated as follows

$$
2 \rho c \int_{0}^{l} y_{t} y_{x} d x \leq \frac{\rho c}{\beta}\left\|y_{t}\right\|^{2}+\beta \rho c\left\|y_{x}\right\|^{2}, \beta>0, t \geq 0 .
$$

The application of Lemma 5 and Lemma 1 to the last two terms in (3.12), leads to

$$
\begin{aligned}
& -\rho c y(l) y_{t}(l) \leq \delta_{1} \rho c l\left\|y_{x}\right\|^{2}+\frac{\rho c}{4 \delta_{1}} y_{t}^{2}(l), \quad \delta_{1}>0, \quad t \geq 0, \\
& m y_{t}(l)\left(y_{t}+c y_{x}\right)(l) \leq m(1+c) y_{t}^{2}(l)+\frac{m c}{4} y_{x}^{2}(l), \quad t \geq 0 .
\end{aligned}
$$

Making use of the estimates (3.13)-(3.15) in (3.12) and applying Lemma 3, we obtain

$$
\begin{aligned}
\frac{d}{d t} \Phi_{1}(t) & \leq \rho\left(1+\frac{c}{\beta}\right)\left\|y_{t}\right\|^{2}-\left[T_{s}-\rho c^{2}-\frac{k}{2}\|a\|_{\infty}-\beta \rho c-\delta_{1} \rho c l\right]\left\|y_{x}\right\|^{2} \\
+ & \frac{1}{2} \int_{0}^{t} h(t-s)\left\|\sqrt{a(x)} y_{x}(s)\right\|^{2} d s-\frac{1}{2} \int_{0}^{l}\left(h \circ y_{x}\right) d x+\frac{m c}{4} y_{x}^{2}(l) \\
+ & {\left[m(1+c)+\frac{\rho c}{4 \delta_{1}}\right] y_{t}^{2}(l)+y(l)\left(F_{d}(t)-d(t)\right), t \geq 0 }
\end{aligned}
$$

For $\Phi_{2}(t)$, we have

$$
\frac{d}{d t} \Phi_{2}(t)=\int_{0}^{l} \frac{d}{d t} \widetilde{\Phi_{2}}(x, t) d x-\frac{d}{d t}\left[m\left(y_{t}+c y_{x}\right)(l) \int_{0}^{t} h(t-s)(y(l, t)-y(l, s)) d s\right],
$$


or

$$
\begin{aligned}
& \frac{d}{d t} \Phi_{2}(t)=\int_{0}^{l}\left(\frac{\partial}{\partial t} \widetilde{\Phi_{2}}(x, t)\right) d x+\left.c \widetilde{\Phi_{2}}(x, t)\right|_{0} ^{l} \\
&-\frac{d}{d t}\left[m\left(y_{t}+c y_{x}\right)(l) \int_{0}^{t} h(t-s)(y(l, t)-y(l, s)) d s\right]
\end{aligned}
$$

where

$$
\widetilde{\Phi_{2}}(x, t)=-\rho y_{t}(x, t) \int_{0}^{t} h(t-s)(y(x, t)-y(x, s)) d s, t \geq 0 .
$$

In view of the boundary conditions in (1.1), we see that

$$
\left.\widetilde{\Phi_{2}}(x, t)\right|_{0} ^{l}=-\rho y_{t}(l) \int_{0}^{t} h(t-s)(y(l, t)-y(l, s)) d s, t \geq 0 .
$$

The relation (3.17), together with (3.18), implies

$$
\begin{aligned}
& \frac{d}{d t} \Phi_{2}(t)=-\rho \int_{0}^{l} y_{t t} \int_{0}^{t} h(t-s)(y(t)-y(s)) d s d x \\
& -\rho \int_{0}^{l} y_{t}\left[\int_{0}^{t} h^{\prime}(t-s)(y(t)-y(s)) d s+\left(\int_{0}^{t} h(s) d s\right) y_{t}\right] d x \\
& \quad-m\left(y_{t t}+c y_{x t}\right)(l) \int_{0}^{t} h(t-s)(y(l, t)-y(l, s)) d s \\
& \quad-m\left(y_{t}+c y_{x}\right)(l)\left[\int_{0}^{t} h^{\prime}(t-s)(y(l, t)-y(l, s)) d s+\left(\int_{0}^{t} h(s) d s\right) y_{t}(l)\right] \\
& \quad-\rho c y_{t}(l) \int_{0}^{t} h(t-s)(y(l, t)-y(l, s)) d s, t \geq 0 .
\end{aligned}
$$

Substituting $y_{t t}$ and $y_{t t}(l)$ from Equation(1.1) into Equation (3.19), integrating by parts and taking into account the boundary conditions and the expression (3.5), it results that

$$
\begin{aligned}
& \frac{d}{d t} \Phi_{2}(t)=\int_{0}^{l}\left(T_{s}-\rho c^{2}-a(x) \int_{0}^{t} h(s) d s\right) y_{x} \int_{0}^{t} h(t-s)\left(y_{x}(t)-y_{x}(s)\right) d s \\
& \quad+\int_{0}^{l} a(x)\left|\int_{0}^{t} h(t-s)\left(y_{x}(t)-y_{x}(s)\right) d s\right|^{2} d x \\
& \quad-\left(\rho \int_{0}^{t} h(s) d s\right)\left\|y_{t}\right\|^{2}-2 \rho c \int_{0}^{l} y_{t} \int_{0}^{t} h(t-s)\left(y_{x}(t)-y_{x}(s)\right) d s d x \\
& \quad-\rho \int_{0}^{l} y_{t} \int_{0}^{t} h^{\prime}(t-s)(y(t)-y(s)) d s d x-m\left(y_{t}+c y_{x}\right)(l) \\
& \quad \times\left[\int_{0}^{t} h^{\prime}(t-s)(y(l, t)-y(l, s)) d s+\left(\int_{0}^{t} h(s) d s\right) y_{t}(l)\right] \\
& \quad+\left(\rho c y_{t}(l)-F_{d}(t)+d(t)\right) \int_{0}^{t} h(t-s)(y(l, t)-y(l, s)) d s, t \geq 0 .
\end{aligned}
$$


For all measurable sets $\mathcal{A}$ and $\mathcal{F}$ such that $\mathcal{A}=\mathbb{R}_{+} \backslash \mathcal{F}$, we have

$$
\begin{aligned}
\int_{0}^{l} & y_{x} \int_{0}^{t} h(t-s)\left(y_{x}(t)-y_{x}(s)\right) d s d x \\
\quad= & \int_{0}^{l} y_{x}\left[\int_{\mathcal{A}_{t}} h(t-s)\left(y_{x}(t)-y_{x}(s)\right) d s+\int_{\mathcal{F}_{t}} h(t-s)\left(y_{x}(t)-y_{x}(s)\right) d s\right] d x \\
\leq & \int_{0}^{l} y_{x} \int_{\mathcal{A}_{t}} h(t-s)\left(y_{x}(t)-y_{x}(s)\right) d s d x+\left(\int_{\mathcal{F}_{t}} h(t-s) d s\right)\left\|y_{x}\right\|^{2} \\
& -\int_{0}^{l} y_{x} \int_{\mathcal{F}_{t}} h(t-s) y_{x}(s) d s d x
\end{aligned}
$$

The first and the last term in the right hand side of (3.21) may be evaluated as follows

$$
\begin{aligned}
& \int_{0}^{l} y_{x} \int_{\mathcal{A}_{t}} h(t-s)\left(y_{x}(s)-y_{x}(t)\right) d s d x \\
& \quad \leq \delta_{2}\left\|y_{x}\right\|^{2}+\frac{k}{4 \delta_{2} a_{0}} \int_{\mathcal{A}_{t}} h(t-s) \int_{0}^{l} a(x)\left(y_{x}(t)-y_{x}(s)\right)^{2} d x d s, \quad \delta_{2}>0
\end{aligned}
$$

and for $t \geq 0$

$$
\int_{0}^{l} y_{x} \int_{\mathcal{F}_{t}} h(t-s) y_{x}(s) d s d x \leq \frac{1}{2}\left(\int_{\mathcal{F}_{t}} h(t-s) d s\right)\left\|y_{x}\right\|^{2}+\frac{1}{2} \int_{\mathcal{F}_{t}} h(t-s)\left\|y_{x}(s)\right\|^{2} d s .
$$

Therefore, (3.21) becomes for $t \geq 0$

$$
\begin{aligned}
\int_{0}^{l} y_{x} & \int_{0}^{t} h(t-s)\left(y_{x}(s)-y_{x}(t)\right) d s d x \\
\leq & \delta_{2}\left\|y_{x}\right\|^{2}+\frac{k}{4 \delta_{2} a_{0}} \int_{\mathcal{A}_{t}} h(t-s) \int_{0}^{l} a(x)\left(y_{x}(t)-y_{x}(s)\right)^{2} d x d s \\
& +\frac{3}{2}\left(\int_{\mathcal{F}_{t}} h(t-s) d s\right)\left\|y_{x}\right\|^{2}+\frac{1}{2} \int_{\mathcal{F}_{t}} h(t-s)\left\|y_{x}(s)\right\|^{2} d s
\end{aligned}
$$

The second term in the right hand side of (3.20) can be handled similarly. Indeed for $\delta_{3}>0$, we see that for $t \geq 0$

$$
\begin{aligned}
& \int_{0}^{l} a(x)\left|\int_{0}^{t} h(t-s)\left(y_{x}(t)-y_{x}(s)\right) d s\right|^{2} d x \\
& \leq\left(1+\frac{1}{\delta_{3}}\right) k \int_{0}^{l} \int_{\mathcal{A}_{t}} h(t-s) a(x)\left(y_{x}(t)-y_{x}(s)\right)^{2} d s d x \\
& \quad+\left(1+\delta_{3}\right) k\left(\int_{\mathcal{F}_{t}} h(t-s) d s\right) \int_{0}^{l} \int_{\mathcal{F}_{t}} h(t-s) a(x)\left(y_{x}(t)-y_{x}(s)\right)^{2} d s d x
\end{aligned}
$$


For the fourth term, we can write for $\delta_{4}>0$ and for $t \geq 0$

$$
\begin{aligned}
& -2 \rho c \int_{0}^{l} y_{t} \int_{0}^{t} h(t-s)\left(y_{x}(t)-y_{x}(s)\right) d s d x \\
& \leq \delta_{4} \rho c\left\|y_{t}\right\|^{2}+\frac{\rho c}{\delta_{4}} \int_{0}^{l}\left|\int_{0}^{t} h(t-s)\left(y_{x}(t)-y_{x}(s)\right) d s\right|^{2} d x
\end{aligned}
$$

or

$$
\begin{aligned}
& -2 \rho c \int_{0}^{l} y_{t} \int_{0}^{t} h(t-s)\left(y_{x}(t)-y_{x}(s)\right) d s d x \\
& \leq \delta_{4} \rho c\left\|y_{t}\right\|^{2}+\frac{2 \rho c}{\delta_{4} a_{0}} k \int_{0}^{l} \int_{\mathcal{A}_{t}} h(t-s) a(x)\left(y_{x}(t)-y_{x}(s)\right)^{2} d s d x \\
& \quad+\frac{2 \rho c}{\delta_{4} a_{0}}\left(\int_{\mathcal{F}_{t}} h(t-s) d s\right) \int_{0}^{l} \int_{\mathcal{F}_{t}} h(t-s) a(x)\left(y_{x}(t)-y_{x}(s)\right)^{2} d s d x
\end{aligned}
$$

In the same manner, we have

$$
\begin{aligned}
& -\int_{0}^{l} y_{t} \int_{0}^{t} h^{\prime}(t-s)(y(t)-y(s)) d s d x \\
& \quad \leq \delta_{5}\left\|y_{t}\right\|^{2}+\frac{l^{2}}{4 \delta_{5} a_{0}}\left(\int_{0}^{t}\left|h^{\prime}(s)\right| d s\right) \int_{0}^{l}\left(\left|h^{\prime}\right| \circ y_{x}\right) d x \\
& \quad \leq \delta_{5}\left\|y_{t}\right\|^{2}-\frac{h(0) l^{2}}{4 \delta_{5} a_{0}} \int_{0}^{l}\left(h^{\prime} \circ y_{x}\right) d x, \delta_{5}>0, \quad t \geq 0 .
\end{aligned}
$$

The remaining terms may be treated for $t \geq 0$, with the help of Lemma 5 and Lemma 1, as follows

$$
\begin{aligned}
& -m\left(y_{t}+c y_{x}\right)(l)\left[\int_{0}^{t} h^{\prime}(t-s)(y(l, t)-y(l, s)) d s\right] \\
& \quad \leq \frac{m}{2}\left(y_{t}^{2}+c^{2} y_{x}^{2}\right)(l)-\frac{m}{a_{0}}\left(\int_{0}^{t}\left|h^{\prime}(s)\right| d s\right)\left(h^{\prime} \circ y\right)(l) \\
& \quad \leq \frac{m}{2}\left(y_{t}^{2}+c^{2} y_{x}^{2}\right)(l)-\frac{\delta_{6} m h(0) l}{a_{0}} \int_{0}^{l}\left(h^{\prime} \circ y_{x}\right) d x \\
& -m\left(\int_{0}^{t} h(s) d s\right)\left(y_{t}+c y_{x}\right)(l) y_{t}(l) \\
& \quad \leq-m\left(\int_{0}^{t} h(s) d s\right) y_{t}^{2}(l)+\frac{m}{2}\left(\int_{0}^{t} h(s) d s\right)\left(y_{t}^{2}+c^{2} y_{x}^{2}\right)(l) \\
& \quad \leq-\frac{m}{2} h_{*} y_{t}^{2}(l)+\frac{m c^{2}}{2} k y_{x}^{2}(l), \quad t \geq t_{*}
\end{aligned}
$$

and

$$
\rho c y_{t}(l) \int_{0}^{t} h(t-s)(y(l, t)-y(l, s)) d s \leq \frac{\rho c}{4 \delta_{7}} y_{t}^{2}(l)+\frac{\delta_{7} \rho c l k}{a_{0}} \int_{0}^{l}\left(h \circ y_{x}\right) d x
$$


for all $t \geq 0$. Collecting the previous estimates (3.22)-(3.28) and inserting them in (3.20), we obtain for $t \geq t_{*}$

$$
\begin{aligned}
& \frac{d}{d t} \Phi_{2}(t) \leq \rho\left(\delta_{5}+\delta_{4} c-h_{*}\right)\left\|y_{t}\right\|^{2}-\frac{h(0) l}{a_{0}}\left(\frac{\rho l}{4 \delta_{5}}+m\right) \int_{0}^{l}\left(h^{\prime} \circ y_{x}\right) d x \\
& +\left(T_{s}-\rho c^{2}-a_{0} h_{*}\right)\left[\delta_{2}+\frac{3}{2}\left(\int_{\mathcal{F}_{t}} h(t-s) d s\right)\right]\left\|y_{x}\right\|^{2} \\
& +k\left[1+\frac{1}{\delta_{3}}+\frac{1}{4 \delta_{2} a_{0}}\left(T_{s}-\rho c^{2}-a_{0} h_{*}\right)+\frac{2 \rho c}{\delta_{4} a_{0}}\right] \\
& \quad \times \int_{0}^{l} \int_{\mathcal{A}_{t}} h(t-s) a(x)\left(y_{x}(t)-y_{x}(s)\right)^{2} d s d x+\frac{1}{2}\left(T_{s}-\rho c^{2}-a_{0} h_{*}\right) \\
& \times \int_{\mathcal{F}_{t}} h(t-s)\left\|y_{x}(s)\right\|^{2} d s+\frac{\delta_{7} \rho c l k}{a_{0}} \int_{0}^{l}\left(h \circ y_{x}\right) d x+\left(1+\delta_{3}+\frac{2 \rho c}{\delta_{4} a_{0}}\right) \\
& \times\left(\int_{\mathcal{F}_{t}} h(t-s) d s\right) \int_{0}^{l} \int_{\mathcal{F}_{t}} h(t-s) a(x)\left(y_{x}(t)-y_{x}(s)\right)^{2} d s d x \\
& +\frac{m c^{2}}{2}\left(k+\frac{1}{\delta_{6}}\right) y_{x}^{2}(l)+\frac{m}{2}\left(-h_{*}+1+\frac{\rho c}{2 \delta_{7} m}\right) y_{t}^{2}(l) \\
& -\left(F_{d}(t)-d(t)\right) \int_{0}^{t} h(t-s)(y(l, t)-y(l, s)) d s .
\end{aligned}
$$

In view of the estimates (3.8), (3.16) and (3.29), we infer that for $t \geq t_{*}$

$$
\begin{aligned}
& \frac{d}{d t} L(t) \leq\left\{\frac{1}{2}-\frac{\lambda_{2} h(0) l}{a_{0}}\left(\frac{\rho l}{4 \delta_{5}}+m\right)\right\} \int_{0}^{l}\left(h^{\prime} \circ y_{x}\right) d x \\
& +\left\{\lambda_{2}\left(T_{s}-\rho c^{2}-a_{0} h_{*}\right)\left(\delta_{2}+2 k \hat{h}(\mathcal{F})\right)\right. \\
& \left.-\lambda_{1}\left[T_{s}-\rho c^{2}-\frac{k}{2}\|a\|_{\infty}-\beta \rho c-\delta_{1} \rho c l\right]\right\}\left\|y_{x}\right\|^{2} \\
& +\rho\left\{\lambda_{1}(1+c / \beta)+\lambda_{2}\left(\delta_{5}+\delta_{4} c-h_{*}\right)\right\}\left\|y_{t}\right\|^{2} \\
& +\left\{\lambda_{2}\left(1+\delta_{3}+\frac{2 \rho c}{\delta_{4} a_{0}}\right) k \hat{h}(\mathcal{F})+\frac{\lambda_{2}}{a_{0}} \delta_{7} \rho c l k-\frac{\lambda_{1}}{2}\right\} \int_{0}^{l}\left(h \circ y_{x}\right) d x \\
& +\frac{\lambda_{1}}{2} \int_{0}^{t} h(t-s)\left\|\sqrt{a(x)} y_{x}(s)\right\|^{2} d s \\
& +\frac{\lambda_{2}}{2 a_{0}}\left(T_{s}-\rho c^{2}-a_{0} h_{*}\right) \int_{\mathcal{F}_{t}} h(t-s)\left\|\sqrt{a(x)} y_{x}(s)\right\|^{2} d s \\
& +\lambda_{2} k\left(1+\frac{1}{\delta_{3}}+\frac{1}{4 \delta_{2} a_{0}}\left(T_{s}-\rho c^{2}-a_{0} h_{*}\right)+\frac{2 \rho c}{\delta_{4} a_{0}}\right) \\
& \times \int_{0}^{l} \int_{\mathcal{A}_{t}} h(t-s) a(x)\left(y_{x}(t)-y_{x}(s)\right)^{2} d s d x+\frac{c}{2} \int_{0}^{t} h(t-s) y_{x}^{2}(l, s) d s \\
& +\left\{\lambda_{2} \frac{m c^{2}}{2}\left(k+\frac{1}{\delta_{6}}\right)+\lambda_{1} \frac{m c}{4}-\frac{c}{2}\left(T_{s}(l)-\rho c^{2}\right)\right\} y_{x}^{2}(l)
\end{aligned}
$$




$$
\begin{aligned}
& +\left\{\lambda_{2} \frac{m}{2}\left(-h_{*}+1+\frac{\rho c}{2 \delta_{7} m}\right)+\lambda_{1}\left[m(1+c)+\frac{\rho c}{4 \delta_{1}}\right]-\frac{\rho c}{2}\right\} y_{t}^{2}(l) \\
& +\left[\left(y_{t}+c y_{x}\right)(l)+\lambda_{1} y(l)-\lambda_{2} \int_{0}^{t} h(t-s)(y(l, t)-y(l, s)) d s\right]\left(F_{d}(t)-d(t)\right) \\
& +\frac{1}{\gamma_{d}} \tilde{\mu}_{d} \frac{d}{d t} \hat{\mu}_{d} .
\end{aligned}
$$

Now, we estimate the last two terms in (3.30). For this, we set

$$
\bar{y}(l)=\left(y_{t}+c y_{x}\right)(l)+\lambda_{1} y(l)-\lambda_{2} \int_{0}^{t} h(t-s)(y(l, t)-y(l, s)) d s
$$

and consider the expressions (3.6) and (3.7), we get

$$
\begin{aligned}
\bar{y}(l)( & \left.F_{d}(t)-d(t)\right)+\frac{1}{\gamma_{d}} \tilde{\mu}_{d} \frac{d}{d t} \hat{\mu}_{d} \\
\leq & -\frac{\hat{\mu}_{d}^{2}(t)}{\hat{\mu}_{d}(t)|\bar{y}(l)|+\varepsilon_{d}}|\bar{y}(l)|^{2}+\mu_{d}|\bar{y}(l)|-\frac{\delta_{d}}{\gamma_{d}} \tilde{\mu}_{d} \hat{\mu}_{d}(t)+\tilde{\mu}_{d}|\bar{y}(l)| \\
\leq & \frac{-\hat{\mu}_{d}^{2}(t)|\bar{y}(l)|^{2}+\hat{\mu}_{d}^{2}(t)|\bar{y}(l)|^{2}+\varepsilon_{d} \hat{\mu}_{d}(t)|\bar{y}(l)|}{\hat{\mu}_{d}(t)|\bar{y}(l)|+\varepsilon_{d}}-\frac{\delta_{d}}{2 \gamma_{d}} \tilde{\mu}_{d}^{2} \\
& -\left(\sqrt{\frac{\delta_{d}}{2 \gamma_{d}}} \tilde{\mu}_{d}+\sqrt{\frac{\delta_{d}}{2 \gamma_{d}}} \mu_{d}\right)^{2}+\frac{\delta_{d}}{2 \gamma_{d}} \mu_{d} \\
\leq & \varepsilon_{d}-\frac{\delta_{d}}{\gamma_{d}} \tilde{\mu}_{d}^{2}+\frac{\delta_{d}}{2 \gamma_{d}} \mu_{d}=\vartheta(t), t \geq 0 .
\end{aligned}
$$

Next, we multiply both sides (3.30) by $e^{2 \sigma s}, 0<\sigma<\lambda / 2$, take into account (3.31) and integrate from $t_{*}$ to $t$ to get

$$
\begin{array}{rl}
e^{2 \sigma t} & L(t)-L\left(t_{*}\right)-2 \sigma \int_{t_{*}}^{t} e^{2 \sigma s} L(s) d s \leq\left\{\frac{1}{2}-\frac{\lambda_{2} h(0) l}{a_{0}}\left(\frac{\rho l}{4 \delta_{5}}+\delta_{6} m\right)\right\} \\
& \times \int_{t_{*}}^{t} e^{2 \sigma s} \int_{0}^{l}\left(h^{\prime} \circ y_{x}\right)(s) d x d s+\left\{\lambda_{2}\left(T_{s}-\rho c^{2}-a_{0} h_{*}\right)\left(\delta_{2}+2 k \hat{h}(\mathcal{F})\right)\right. \\
& \left.-\lambda_{1}\left[T_{s}-\rho c^{2}-\frac{k}{2}\|a\|_{\infty}-\beta \rho c-\delta_{1} \rho c l\right]\right\} \int_{t_{*}}^{t} e^{2 \sigma s}\left\|y_{x}\right\|^{2} d s+\rho\left\{\lambda_{1}\left(1+\frac{c}{\beta}\right)\right. \\
+ & \left.\lambda_{2}\left(\delta_{5}+\delta_{4} c-h_{*}\right)\right\} \int_{t_{*}}^{t} e^{2 \sigma s}\left\|y_{t}\right\|^{2} d s \\
+ & \left\{\lambda_{2}\left(1+\delta_{3}+\frac{2 \rho c}{\delta_{4} a_{0}}\right) k \hat{h}(\mathcal{F})+\frac{\lambda_{2}}{a_{0}} \delta_{7} \rho c l k-\frac{\lambda_{1}}{2}\right\} \int_{t_{*}}^{t} e^{2 \sigma s} \int_{0}^{l}\left(h \circ y_{x}\right)(s) d x d s \\
+ & \frac{\lambda_{1}}{2} \int_{t_{*}}^{t} e^{2 \sigma s} \int_{0}^{s} h(s-z)\left\|\sqrt{a(x)} y_{x}(z)\right\|^{2} d z d s \\
+ & \frac{\lambda_{2}}{2 a_{0}}\left(T_{s}-\rho c^{2}-a_{0} h_{*}\right) \int_{t_{*}}^{t} e^{2 \sigma s} \int_{\mathcal{F}_{s}} h(s-z)\left\|\sqrt{a(x)} y_{x}(z)\right\|^{2} d z d s
\end{array}
$$




$$
\begin{aligned}
& +\lambda_{2} k\left(1+\frac{1}{\delta_{3}}+\frac{1}{4 \delta_{2} a_{0}}\left(T_{s}-\rho c^{2}-a_{0} h_{*}\right)+\frac{2 \rho c}{\delta_{4} a_{0}}\right) \\
& +\int_{t_{*}}^{t} e^{2 \sigma s} \int_{0}^{l} \int_{\mathcal{A}_{s}} h(t-s)\left(y_{x}(s)-y_{x}(z)\right)^{2} d z d x d s \\
& +\left\{\frac{\lambda_{2}}{2} m c^{2}\left(k+\frac{1}{\delta_{6}}\right)+\lambda_{1} \frac{m c}{4}-\frac{c}{2}\left(T_{s}(l)-\rho c^{2}\right)\right\} \int_{t_{*}}^{t} e^{2 \sigma s} y_{x}^{2}(l, s) d s \\
& +\left\{\lambda_{2} m\left[-\frac{h_{*}}{2}+\frac{1}{2 \delta_{6}}+\frac{\rho c}{4 \delta_{7} m}\right]+\lambda_{1}\left[m(1+c)+\frac{\rho c}{4 \delta_{1}}\right]-\frac{\rho c}{2}\right\} \int_{t_{*}}^{t} e^{2 \sigma s} y_{t}^{2}(l, s) d s \\
& +\frac{c}{2} \int_{t_{*}}^{t} e^{2 \sigma s} \int_{0}^{s} h(s-z) y_{x}^{2}(l, z) d z d s+\int_{t_{*}}^{t} e^{2 \sigma s} \vartheta(s) d s, t \geq t_{*} .
\end{aligned}
$$

For $\beta<\frac{1}{\rho c}\left(T_{s}-\rho c^{2}-\frac{k}{2}\|a\|_{\infty}\right)$, we choose $\delta_{4}=\frac{h_{*}}{4 c}, \delta_{5}=\frac{h_{*}}{4}$ and $\lambda_{1}=$ $\frac{\beta h_{*}}{4(\beta+c)} \lambda_{2}$. We need

$$
\frac{1}{2}-\frac{\lambda_{2} h(0) l}{a_{0}}\left(\frac{\rho l}{4 \delta_{5}}+m\right) \geq \frac{1}{4} .
$$

This is possible if we select

$$
\lambda_{2}<a_{0} h_{*} /\left[4 h(0) l\left(\rho l+h_{*} m\right)\right] .
$$

We use Lemma 2 and Lemma 4 to get

$$
\begin{aligned}
\int_{t_{*}}^{t} e^{2 \sigma s} \int_{0}^{s} h(s-z)\left\|\sqrt{a(x)} y_{x}(z)\right\|^{2} d z d s \\
\quad=\int_{t_{*}}^{t} \int_{0}^{s} h(s-z) e^{2 \sigma(s-z)} e^{2 \sigma z}\left\|\sqrt{a(x)} y_{x}(z)\right\|^{2} d z d s \\
\leq \int_{t_{*}}^{t} h(s) e^{2 \sigma s} d s \int_{t_{*}}^{t} e^{2 \sigma s}\left\|\sqrt{a(x)} y_{x}(z)\right\|^{2} d s \\
\leq(1+\varepsilon) k \int_{t_{*}}^{t} e^{2 \sigma s}\left\|\sqrt{a(x)} y_{x}(z)\right\|^{2} d s \\
\leq(1+\varepsilon) k\|a\|_{\infty} \int_{t_{*}}^{t} e^{2 \sigma s}\left\|y_{x}(z)\right\|^{2} d s, t \geq t_{*}
\end{aligned}
$$

and

$$
\begin{aligned}
& \int_{t_{*}}^{t} e^{2 \sigma s} \int_{0}^{s} h(s-z) y_{x}^{2}(l, z) d z d s=\int_{t_{*}}^{t} \int_{0}^{s} h(s-z) e^{2 \sigma(s-z)} e^{2 \sigma z} y_{x}^{2}(l, z) d z d s \\
& \leq \int_{t_{*}}^{t} h(s) e^{2 \sigma s} d s \int_{t_{*}}^{t} e^{2 \sigma s} y_{x}^{2}(l, s) d s \leq(1+\varepsilon) k \int_{t_{*}}^{t} e^{2 \sigma s} y_{x}^{2}(l, s) d s, t \geq t_{*} .
\end{aligned}
$$

Next, in view of Proposition 1, we find

$$
\begin{aligned}
& 2 \sigma \int_{t_{*}}^{t} e^{2 \sigma s} L(s) d s \leq 4 \sigma \int_{t_{*}}^{t} e^{2 \sigma s} E(s) d s \\
& \quad \leq 2 \sigma \int_{t_{*}}^{t} e^{2 \sigma s}\left\{\rho\left\|y_{t}\right\|^{2}+\left[T_{s}-\rho c^{2}-a_{0} h_{*}+2 k(2+\varepsilon)\right]\left\|y_{x}\right\|^{2}\right\} d s
\end{aligned}
$$




$$
+4 \sigma m \int_{t_{*}}^{t} e^{2 \sigma s}\left(y_{t}^{2}(l)+c^{2} y_{x}^{2}(l)\right) d s, \quad t \geq t_{*} .
$$

From (3.32) and with the help of the relations (3.33), (3.34) and (3.35), we infer that

$$
\begin{array}{rl}
e^{2 \sigma t} & L(t)-L\left(t_{*}\right) \leq \frac{1}{4} \int_{0}^{t} e^{2 \sigma s} \int_{0}^{l}\left(h^{\prime} \circ y_{x}\right)(s) d x d s \\
+ & \left\{\lambda_{2}\left(T_{s}-\rho c^{2}-a_{0} h_{*}\right)\left(\delta_{2}+2 k \hat{h}(\mathcal{F})\right)\right. \\
- & \lambda_{1}\left[T_{s}-\rho c^{2}-\left(1+\frac{\varepsilon}{2}\right) k\|a\|_{\infty}-\beta \rho c-\delta_{1} \rho c l\right] \\
+ & \left.2 \sigma\left[T_{s}-\rho c^{2}-a_{0} h_{*}+2 k(2+\varepsilon)\right]\right\} \int_{t_{*}}^{t} e^{2 \sigma s}\left\|y_{x}\right\|^{2} d s \\
+ & \left(2 \sigma-\lambda_{2} \frac{\rho h_{*}}{4}\right) \int_{t_{*}}^{t} e^{2 \sigma s}\left\|y_{t}\right\|^{2} d s \\
+ & \left\{\lambda_{2}\left(1+\delta_{3}+\frac{2 \rho c}{\delta_{4} a_{0}}\right) k \hat{h}(\mathcal{F})+\frac{\lambda_{2}}{a_{0}} \delta_{7} \rho c l k-\frac{\lambda_{1}}{2}\right\} \int_{t_{*}}^{t} e^{2 \sigma s} \int_{0}^{l}\left(h \circ y_{x}\right)(s) d x d s \\
+ & \lambda_{2} k\left[1+\frac{1}{\delta_{3}}+\frac{1}{4 \delta_{2}}\left(T_{s, \max }-\rho c^{2}-h_{*}\right)+\frac{2 \rho c}{\delta_{4}}\right] \\
& \times \int_{t_{*}}^{t} e^{2 \sigma s} \int_{0}^{l} \int_{\mathcal{A}_{s}} h(t-s)\left(y_{x}(s)-y_{x}(z)\right)^{2} d z d x d s \\
+ & \left\{\lambda_{2} \frac{m c^{2}}{2}\left(k+\frac{1}{\delta_{6}}\right)+\lambda_{1} \frac{m c}{4}-\frac{c}{2}\left[T_{s}(l)-\rho c^{2}-(1+\varepsilon) k\right]+4 \sigma m c^{2}\right\} \\
+ & \left.\lambda_{1}\left[m(1+c)+\frac{\rho c}{4 \delta_{1}}\right]-\frac{\rho c}{2}\right\} \times \int_{t_{*}}^{t} e^{2 \sigma s} y_{t}^{2}(l, s) d s+\int_{t_{*}}^{t} e^{2 \sigma s} \vartheta(s) d s, t \geq t_{*} \cdot \\
& \times \int_{t_{*}}^{t} e^{2 \sigma s} y_{x}^{2}(l, s) d s+\left\{\lambda_{2} m\left[-\frac{h_{*}}{2}+\frac{1}{2}+\frac{\rho c}{4 \delta_{7} m}\right]\right.
\end{array}
$$

As in [31], we introduce

$$
\begin{aligned}
& \mathcal{A}_{n}=\left\{s \in \mathbb{R}_{+}: n h^{\prime}(s)+h(s) \leq 0\right\}, \quad n \in \mathbb{N}, \\
& \tilde{A}_{n t}=\left\{s \in \mathbb{R}_{+}: 0 \leq s \leq t, n h^{\prime}(t-s)+h(t-s) \leq 0\right\}, \quad n \in \mathbb{N} .
\end{aligned}
$$

Observe that $\bigcup_{n} \mathcal{A}_{n}=\mathbb{R}_{+} \backslash\left\{\mathcal{F}_{h} \cup \mathcal{N}_{h}\right\}$, where $\mathcal{N}_{h}$ is the set where $h^{\prime}$ is not defined and $\mathcal{F}_{h}$ is defined in (2.1), if we denote $\mathcal{F}_{n}=\mathbb{R}_{+} \backslash \mathcal{A}_{n}$, then

$$
\lim _{n \rightarrow \infty} \hat{h}\left(\mathcal{F}_{n}\right)=\hat{h}\left(\mathcal{F}_{h}\right)
$$

because $\mathcal{F}_{n+1} \subset \mathcal{F}_{n}$ for all $n$ and $\bigcap_{n} \mathcal{F}_{n}=\mathcal{F}_{h} \cup \mathcal{N}_{h}$. We take $\mathcal{A}_{t}:=\tilde{A}_{n t}$, $\mathcal{F}_{t}:=\tilde{F}_{n t}$ in (3.36). We get

$$
\begin{gathered}
e^{2 \sigma t} L(t)-L\left(t_{*}\right) \leq\left\{\lambda_{2}\left(T_{s}-\rho c^{2}-a_{0} h_{*}\right)\left(\delta_{2}+2 k \hat{h}\left(\mathcal{F}_{n}\right)\right)\right. \\
-\lambda_{1}\left[T_{s}-\rho c^{2}-\left(1+\frac{\varepsilon}{2}\right) k\|a\|_{\infty}-\beta \rho c-\delta_{1} \rho c l\right]
\end{gathered}
$$




$$
\begin{aligned}
+ & \left.2 \sigma\left[T_{s}-\rho c^{2}-a_{0} h_{*}+2 k(2+\varepsilon)\right]\right\} \times \int_{t_{*}}^{t} e^{2 \sigma s}\left\|y_{x}\right\|^{2} d s \\
+ & \left(2 \sigma-\lambda_{2} \frac{\rho h_{*}}{4}\right) \int_{t_{*}}^{t} e^{2 \sigma s}\left\|y_{t}\right\|^{2} d s \\
+ & \left\{\lambda_{2}\left(1+\delta_{3}+\frac{2 \rho c}{\delta_{4} a_{0}}\right) k \hat{h}\left(\mathcal{F}_{n}\right)+\frac{\lambda_{2}}{a_{0}} \delta_{7} \rho c l k-\frac{\lambda_{1}}{2}\right\} \\
& \times \int_{t_{*}}^{t} e^{2 \sigma s} \int_{0}^{l}\left(h \circ y_{x}\right)(s) d x d s \\
+ & \left\{\lambda_{2} k\left[1+\frac{1}{\delta_{3}}+\frac{1}{4 \delta_{2}}\left(T_{s}-\rho c^{2}-h_{*}\right)+\frac{2 \rho c}{\delta_{4}}\right]-\frac{1}{4 n}\right\} \\
& \times \int_{t_{*}}^{t} e^{2 \sigma s} \int_{0}^{l} \int_{\mathcal{A}_{n s}} h(t-s)\left(y_{x}(s)-y_{x}(z)\right)^{2} d z d x d s \\
+ & \left\{\lambda_{2} \frac{m c^{2}}{2}(k+1)+\lambda_{1} \frac{m c}{4}-\frac{c}{2}\left[T_{s}(l)-\rho c^{2}-(1+\varepsilon) k\right]+4 \sigma m c^{2}\right\} \\
& \times \int_{t_{*}}^{t} e^{2 \sigma s} y_{x}^{2}(l, s) d s+\left\{\lambda_{2} m\left[-\frac{h_{*}}{2}+\frac{1}{2 \delta_{6}}+\frac{\rho c}{4 \delta_{7} m}\right]+\lambda_{1}\left[m(1+c)+\frac{\rho c}{4 \delta_{1}}\right]\right. \\
- & \left.\frac{\rho c}{2}+4 \sigma m\right\} \int_{t_{*}}^{t} e^{2 \sigma s} y_{t}^{2}(l, s) d s+\int_{t_{*}}^{t} e^{2 \sigma s} \vartheta(s) d s, \quad t \geq t_{*} .
\end{aligned}
$$

For simplicity, we take $\delta_{2}=\delta_{3}$ and $\delta_{7}=\frac{a_{0} \beta}{16(\beta+c) \rho c l k} h_{*}$.

For $\beta<\frac{1}{\rho c}\left(T_{s}-\rho c^{2}-k\|a\|_{\infty}\right)$, we choose

$$
\delta_{1}=\frac{1}{2 \rho c l}\left[T_{s}-\rho c^{2}-\left(1+\frac{\varepsilon}{2}\right) k\|a\|_{\infty}-\beta \rho c\right] .
$$

Now, we start selecting the different parameters in such a way that all the coefficients in the right-hand side of (3.37) are negative. For this, we set

$$
\left\{\begin{aligned}
k_{1}= & \left(T_{s}-\rho c^{2}-a_{0} h_{*}\right)\left(\delta_{2}+2 k \hat{h}\left(\mathcal{F}_{n}\right)\right) \\
& -\frac{\beta h_{*}}{8(\beta+c)}\left[T_{s}-\rho c^{2}-\left(1+\frac{\varepsilon}{2}\right) k\|a\|_{\infty}-\beta \rho c\right], \\
k_{2}= & -\lambda_{2} \frac{\rho h_{*}}{4}<0 \\
k_{3}= & \left(1+\delta_{2}+\frac{2 \rho c}{\delta_{4} a_{0}}\right) k \hat{h}\left(\mathcal{F}_{n}\right)-\frac{\beta h_{*}}{16(\beta+c)}, \\
k_{4}= & \lambda_{2} k\left[1+\frac{1}{\delta_{3}}+\frac{1}{4 \delta_{2}}\left(T_{s}-\rho c^{2}-h_{*}\right)+\frac{2 \rho c}{\delta_{4}}\right]-\frac{1}{4 n}, \\
k_{5}= & \lambda_{2} \frac{m c^{2}}{2}(k+1)+\lambda_{1} \frac{m c}{4}-\frac{c}{2}\left[T_{s}(l)-\rho c^{2}-(1+\varepsilon) k\right], \\
k_{6}= & \lambda_{2} m\left[-\frac{h_{*}}{2}+\frac{1}{2 \delta_{6}}+\frac{\rho c}{4 \delta_{7} m}\right]+\lambda_{1}\left[m(1+c)+\frac{\rho c}{4 \delta_{1}}\right]-\frac{\rho c}{2} .
\end{aligned}\right.
$$

For $k_{1}$ and $k_{3}$, we select $\hat{h}\left(\mathcal{F}_{n}\right)$ small in such that

$$
\left\{\begin{array}{l}
2\left(T_{s}-\rho c^{2}-a_{0} h_{*}\right) k \hat{h}\left(\mathcal{F}_{n}\right)<\frac{\beta h_{*}}{8(\beta+c)}\left(T_{s}-\rho c^{2}-k\|a\|_{\infty}-\beta \rho c\right), \\
\left(1+\frac{2 \rho c}{\delta_{4} a_{0}}\right) k \hat{h}\left(\mathcal{F}_{n}\right)<\frac{\beta}{16(\beta+c)} h_{*} .
\end{array}\right.
$$

Once this is fixed, and in order to make $k_{1}$ and $k_{3}$ negative, we can choose $\delta_{2}$ and $\varepsilon$ small enough. For $k_{i}, i=4, \ldots, 6$, it suffices to take $\lambda_{2}$ small enough such 
that the relation in Proposition 1 remains valid and

$$
\left\{\begin{array}{l}
\lambda_{2} k\left[1+\frac{1}{\delta_{3}}+\frac{1}{4 \delta_{2}}\left(T_{s}-\rho c^{2}-h_{*}\right)+\frac{2 \rho c}{\delta_{4}}\right]<\frac{1}{4 n}, \\
\lambda_{2} \frac{m c^{2}}{2}(k+1)+\lambda_{2} \frac{\beta h_{*}}{4(\beta+c)} \frac{m c}{4}<\frac{c}{2}\left[T_{s}(l)-\rho c^{2}-(1+\varepsilon) k\right], \\
\lambda_{2} m\left[-\frac{h_{*}}{2}+\frac{1}{2 \delta_{6}}+\frac{\rho c}{4 \delta_{7} m}\right]+\lambda_{2} \frac{\beta h_{*}}{4(\beta+c)}\left[m(1+c)+\frac{\rho c}{4 \delta_{1}}\right]<\frac{\rho c}{2} .
\end{array}\right.
$$

To conclude, we choose

$$
\sigma<\min \left\{-\lambda_{2} k_{1} /\left\{2 \sigma\left[T_{s}-\rho c^{2}-a_{0} h_{*}+2 k(2+\varepsilon)\right]\right\},-k_{2},-k_{5} / 4 m c^{2},-k_{6} / 4 m\right\},
$$

which makes negative all the coefficients in (3.37). This leads to

$$
L(t) \leq e^{-2 \sigma t}\left(L\left(t_{*}\right)+\int_{t_{*}}^{t} e^{2 \sigma s} \vartheta(s) d s\right) .
$$

Note that $\vartheta$ is bounded $\left(|\vartheta(t)| \leq \vartheta_{d}, t \geq t_{*}\right)$ because of the assumption that $\mu_{d}$ is bounded (see [34]). This allows us to get

$$
L(t) \leq L\left(t_{*}\right) e^{-2 \sigma t}+\frac{\vartheta_{d}}{2 \sigma}, t \geq t_{*}
$$

Proposition 1 permits to get

$$
E(t) \leq 2 L\left(t_{*}\right) e^{-2 \sigma t}+\frac{\vartheta_{d}}{\sigma}, t \geq t_{*} .
$$

The result holds trivially between 0 and $t_{*} \cdot \vartheta(t)$ can be pushed in an arbitrarily small boundedness region by making sufficiently small $\varepsilon_{d}, \delta_{d}$ and sufficiently large $\gamma_{d}$. (see [33] and [34]). Thus, (3.38) becomes

$$
E(t) \leq C_{1} e^{-\gamma t}+\epsilon_{1}, t \geq 0
$$

with small $\epsilon_{1}$ and positive constants $C_{1}$ and $\gamma$, which implies by applying the Cauchy-Schwarz inequality

$$
|y(x, t)| \leq \sqrt{l}\left\|y_{x}\right\| \leq\left(\frac{2 l E(t)}{\left(T_{s}-\rho c^{2}-\|a\|_{\infty} k\right)}\right)^{\frac{1}{2}} \leq \sqrt{C e^{-\gamma t}+\epsilon}, t \geq 0
$$

with small $\epsilon$ and positive constant $C$. In the case where there is no disturbance, that is $d(t)=0, t \geq 0$, we get the following exponential decay result. Indeed, there exist positive constants $K$ and $\tau$ such that

$$
E(t) \leq K e^{-\tau t}, t \geq 0
$$

Remark 2. Some recent literatures have investigated the boundary control of axially moving systems with axial variable speed $c(t)>0$, (see [24]). Under this assumption, the main equation becomes

$\rho\left(y_{t t}+2 c(t) y_{x t}+c^{2}(t) y_{x x}+a_{v}(t) y_{x}\right)-T_{s} y_{x x}+\int_{0}^{t} h(t-s)\left(a(x) y_{x}(s)\right)_{x} d s=0$, 
where $a_{v}(t)>0$ is the acceleration. The constant speed is a special case of variable speed $\left(a_{v}(t)=0\right)$ and its derivation will be zero. In order to accommodate the terms resulted from the effect of a variable speed, the authors in ( $[24])$ considered a frictional damping term $\left(K\left(y_{t}+v y_{x}\right), K>0\right)$. This is not the case in our work as the dissipation produced by the viscoelastic term is weaker than the one produced by the frictional damping and the new terms could not be easily controlled. In other hand, the aim in this work is to study the effect of viscoelastic materials on the theory of moving structures and its role in the reducing of the undesirable vibrations.

Remark 3. Note that if the inequality in (A2) is strict (which is the case in many existing papers in viscoelasticity), then there will be no restriction on the size of $\mathcal{R}_{h}$. In case of power-type decay of the kernel $h$ we can obtain power-type decay of the solution (see [30]). In most of the existing works a rate of decay is imposed to the kernel through the condition $h^{\prime}(t) \leq-C h(t)$ for some positive constant $C$. This constant can be replaced by a function $\psi(t)$ and the function $h(t)$ can be replaced by a function of $h(t)$ (that is $h^{\prime}(t) \leq$ $-\psi(t) \phi(h(t)))$. It is clear that this condition in its simplest form $h^{\prime}(t) \leq-C h(t)$ imply the assumption in (A3). But, the assumption (A3) does not imply this condition.

\section{References}

[1] S. Abrate. Vibration of belts and belt drives. Mechanism and Machine Theory, 27(6):645-695, 1992. https://doi.org/10.1016/0094-114X(92)90064-O.

[2] A. Berkani, N.e. Tatar and A. Kelleche. Vibration control of a viscoelastic translational Euler-Bernoulli beam. J. Dyn. Control Sys., pp. 1-33, 2017. https://doi.org/10.1007/s10883-017-9364-9.

[3] M.M. Cavalcanti, V.N. Domingos Cavalcanti and J. Ferreira. Existence and uniform decay for a non-linear viscoelastic equation with strong damping. Math. Meth. Appl. Sci, 24(14):1043-1053, 2001. https://doi.org/10.1002/mma.250.

[4] M.M. Cavalcanti, V.N. Domingos Cavalcanti, J.S. Prates Filho and J.A. Soriano. Existence and uniform decay rates for viscoelastic problems with nonlinear boundary damping. Diff. Integral Equ, 14(1):85-116, 2001.

[5] C.H. Chung and C.A. Tan. Active vibration control of the axially moving string by wave cancellation. J. Vib. Acoust., 117(1):49-55, 1995. https://doi.org/10.1115/1.2873866.

[6] B.D. Coleman and V.J. Mizel. On the general theory of fading memory. Arch. Ration. Mech. Anal., 29(1):18-31, 1968. https://doi.org/10.1007/BF00256456.

[7] B.D. Coleman and W. Noll. Foundations of linear viscoelasticity. Rev. Modern. Phys., 33:239-249, 1961. https://doi.org/10.1103/RevModPhys.33.239.

[8] C.M. Dafermos. Asymptotic stability in viscoelasticity. Arch. Ration. Mech. Anal., 37(4):297-308, 1970. https://doi.org/10.1007/BF00251609.

[9] C.M. Dafermos. On abstract Volterra equations with applications to linear viscoelasticity. J. Differential Equations, 7:554-569, 1970. https://doi.org/10.1016/0022-0396(70)90101-4. 
[10] M. Fabrizio and A. Morro. Mathematical Problems in Linear Viscoelasticity. SIAM Stud. Appl. Math. Philadelphia, 1992. https://doi.org/10.1137/1.9781611970807.

[11] R.F. Fung and C.C. Tseng. Boundary control of an axially moving string via Lyapunov method. J. Dyn. Syst. Measurement Control, 121(1):105-110, 1999. https://doi.org/10.1115/1.2802425.

[12] R.F. Fung, J.W. Wu, and S.L. Wu. Exponential stabilization of an axially moving string by linear boundary feedback. Automatica, 35(1):177-181, 1999. https://doi.org/10.1016/S0005-1098(98)00173-3.

[13] G.H. Hardy, J.E. Littlewood and G. Polya. Inequalities. Cambridge, UK: Cambridge University Press, 1959.

[14] Y.H. Kang, J.Y. Park and J.A. Kim. A memory type boundary stabilization for an Euler-Bernoulli beam under boundary output feedback control. J. Korean Math. Soc., 49(5):947-964, 2012. https://doi.org/10.4134/JKMS.2012.49.5.947.

[15] A. Kelleche, A. Berkani and N.e. Tatar. Uniform stabilization of a nonlinear axially moving string by a boundary control of memory type. J. Dyn. Control Sys., 2017. https://doi.org/10.1007/s10883-017-9370-y.

[16] A. Kelleche and N.e. Tatar. Control of an axially moving viscoelastic Kirchhoff string. J. Appl. Anal., 2017. https://doi.org/10.1080/00036811.2016.1277708.

[17] A. Kelleche and N.e. Tatar. Uniform decay for solutions of an axially moving viscoelastic beam. Appl. Maths. Optim., 75(3):343-364, 2017. https://doi.org/10.1007/s00245-016-9334-8.

[18] A. Kelleche, N.e. Tatar and A. Khemmoudj. Stability of an axially moving viscoelastic beam. J. Dyn. Control Sys., 23(2):283-299, 2017. https://doi.org/10.1007/s10883-016-9317-8.

[19] A. Kelleche, N.e. Tatar and A. Khemmoudj. Uniform stabilization of an axially moving Kirchhoff string by a boundary control of memory type. J. Dyn. Control Sys., 23(2):237-247, 2017. https://doi.org/10.1007/s10883-016-9310-2.

[20] L.G. Leal. Advanced transport phenomena: fluid mechanics and connective transport processes. Cambridge University Press, 2007. ISBN: 9780521179089.

[21] S.Y. Lee and C.D. Mote. Vibration control of an axially moving string by boundary control. J. Dyn. Systems Measurement Control, 118(1):66-74, 1996. https://doi.org/10.1115/1.2801153.

[22] Y. Li, D. Aron and C.D. Rahn. Adaptive vibration isolation for axially moving strings:theory and experiment. Automatica, 38(3):379-390, 2002. https://doi.org/10.1016/S0005-1098(01)00219-9.

[23] Y. Liu, B. Xu, Y. Wu and Y. Hu. Boundary control of an axially moving belt. Proceedings of the 32nd Chinese Control Conference, pp. 1310-1315, 2013.

[24] Y. Liu, Z. Zhao and W. He. Boundary control of an axially moving accelerated/decelerated belt system. Inter. J. Robust Non. Control, 26(17):38493866, 2016. https://doi.org/10.1002/rnc.3538.

[25] C.D. Mote. Dynamic stability of axially moving materials. Shock. Vib. Dig., 4(4):2-11, 1972.

[26] F. Odeh and I. Tadjbakhsh. Uniqueness in the linear theory of viscoelasticity. Arch. Ration. Mech. Anal., 18(3):244-250, 1965. 
[27] V. Pata. Exponential stability in linear viscoelasticity. Quart. Appl. Math., 64(3):499-513, 2006. https://doi.org/10.1090/S0033-569X-06-01010-4.

[28] O. Reynolds. Papers on Mechanical and Physical studies. The Sub-Mechanics of the Universe, Cambridge University Press, 1903.

[29] S.M. Shahruz. Boundary control of the axially moving Kirchhoff string. Automatica, 34(10):1273-1277, 1998. https://doi.org/10.1016/S0005-1098(98)00074-0.

[30] N.e. Tatar. Polynomial stability without polynomial decay of the relaxation function. Math. Meth. Appl. Sci., 31(15):1874-1886, 2008. https://doi.org/10.1002/mma.1018.

[31] N.e. Tatar. On a large class of kernels yielding exponential stability in viscoelasticity. Appl. Math. Comp., 215(6):2298-2306, 2009. https://doi.org/10.1016/j.amc.2009.08.034.

[32] N.e. Tatar. A new class of kernels leading to an arbitrary decay in viscoelasticity. Meditter. J. Math., 10(1):213-226, 2013. https://doi.org/10.1007/s00009-0120177-5.

[33] K.J. Yang, K.S. Hong and F. Matsuno. Robust adaptive boundary control of an axially moving string under a spatiotemporally varying tension. J. Sound. Vib., 273(4-5):1007-1029, 2004. https://doi.org/10.1016/S0022-460X(03)00519-4.

[34] K.J. Yang, K.S. Hong and F. Matsuno. Robust adaptive control of a cantilevered flexible structure with spatiotemporally varying coefficients and bounded disturbance. JSME Int. J. Ser. C, 47(3):812-822, 2004. https://doi.org/10.1299/jsmec.47.812. 